Acta Crystallographica Section E

Structure Reports

Online

ISSN 1600-5368

\section{(E)-2-(4-Methoxyphenyl)-N-(2-pyridyl)-3- (2-pyridylamino)acrylamide}

\section{Zhu-Ping Xiao,* Xiao-Chun Peng and Ying-Chun Wang}

College of Chemistry \& Chemical Engineering, Jishou University, Jishou 416000 People's Republic of China

Correspondence e-mail: xiaozhuping2005@163.com

Received 25 February 2009; accepted 25 February 2009

Key indicators: single-crystal X-ray study; $T=293 \mathrm{~K}$; mean $\sigma(\mathrm{C}-\mathrm{C})=0.007 \AA$; $R$ factor $=0.083 ; w R$ factor $=0.200 ;$ data-to-parameter ratio $=15.0$.

In the title compound, $\mathrm{C}_{20} \mathrm{H}_{18} \mathrm{~N}_{4} \mathrm{O}_{2}$, the aminoacrylamide group makes a dihedral angles of $4.0(1)^{\circ}$ with the aminobound pyridyl ring and $15.66(12)^{\circ}$ with the amide-bound pyridyl ring. The dihedral angle between the aminoacrylamide group and the pendant 4-methoxyphenyl group is $71.22(9)^{\circ}$. In the crystal structure, $\mathrm{N}-\mathrm{H} \cdots \mathrm{N}$ hydrogen bonds and $\mathrm{C}-$ $\mathrm{H} \cdots \mathrm{O}$ and $\mathrm{C}-\mathrm{H} \cdots \mathrm{N}$ interactions help to establish the packing. Intramolecular $\mathrm{C}-\mathrm{H} \cdots \mathrm{O}$ and $\mathrm{C}-\mathrm{H} \cdots(\mathrm{N}, \mathrm{O})$ contacts also occur.

\section{Related literature}

For background to the antibacteriological activity of enamines, see: Xiao et al. $(2007,2008)$.<smiles>COc1ccc(/C(=C\Nc2ccccn2)C(=O)Nc2ccccn2)cc1</smiles>

\section{Experimental}

Crystal data

$\mathrm{C}_{20} \mathrm{H}_{18} \mathrm{~N}_{4} \mathrm{O}_{2}$

$M_{r}=346.38$

Monoclinic, $P 2_{1} / c$

$$
\begin{aligned}
& b=12.148(2) \AA \\
& c=14.006(3) \AA \\
& \beta=113.74(3) \circ \\
& V=1798.3(6) \AA^{3} \\
& Z=4
\end{aligned}
$$

Data collection

Enraf-Nonius CAD-4 diffractometer

Absorption correction: $\psi$ scan (North et al., 1968)

$T_{\min }=0.975, T_{\max }=0.992$

3523 measured reflections

Refinement

$R\left[F^{2}>2 \sigma\left(F^{2}\right)\right]=0.083$

$w R\left(F^{2}\right)=0.200$

$S=1.04$

3523 reflections $\mu=0.09 \mathrm{~mm}^{-1}$ $T=293 \mathrm{~K}$ $0.30 \times 0.10 \times 0.10 \mathrm{~mm}$

3523 independent reflections 1452 reflections with $I>2 \sigma(I)$

3 standard reflections every 200 reflections intensity decay: none
Mo $K \alpha$ radiation

Table 1

Hydrogen-bond geometry $\left(\AA{ }^{\circ}\right)$.

\begin{tabular}{lllll}
\hline$D-\mathrm{H} \cdots A$ & $D-\mathrm{H}$ & $\mathrm{H} \cdots A$ & $D \cdots A$ & $D-\mathrm{H} \cdots A$ \\
\hline $\mathrm{N} 1-\mathrm{H} 1 A \cdots \mathrm{N} 4{ }^{\mathrm{i}}$ & 0.86 & 2.25 & $3.079(5)$ & 163 \\
$\mathrm{C} 9-\mathrm{H} 9 A \cdots \mathrm{O} 2$ & 0.93 & 2.33 & $2.718(5)$ & 104 \\
$\mathrm{C} 9-\mathrm{H} 9 A \cdots \mathrm{N} 2$ & 0.93 & 2.42 & $2.754(6)$ & 101 \\
$\mathrm{C} 17-\mathrm{H} 17 A \cdots \mathrm{O} 2$ & 0.93 & 2.27 & $2.850(5)$ & 120 \\
$\mathrm{C} 11-\mathrm{H} 11 A \cdots \mathrm{N} 4{ }^{\mathrm{i}}$ & 0.93 & 2.62 & $3.396(6)$ & 141 \\
$\mathrm{C} 14-\mathrm{H} 14 A \cdots \mathrm{O} 2^{\text {ii }}$ & 0.93 & 2.59 & $3.378(5)$ & 143 \\
\hline
\end{tabular}

Symmetry codes: (i) $-x+2, y-\frac{1}{2},-z+\frac{1}{2}$; (ii) $-x+1, y-\frac{1}{2},-z+\frac{1}{2}$.

Data collection: CAD-4 Software (Enraf-Nonius, 1989); cell refinement: $C A D-4$ Software; data reduction: XCAD4 (Harms \& Wocadlo, 1995); program(s) used to solve structure: SHELXS97 (Sheldrick, 2008); program(s) used to refine structure: SHELXL97 (Sheldrick, 2008); molecular graphics: SHELXTL (Sheldrick, 2008); software used to prepare material for publication: SHELXL97.

The work was financed by a grant (No. JSDXKYZZ0801) for talent introduction from Jishou University, China.

Supplementary data and figures for this paper are available from the IUCr electronic archives (Reference: HB2919).

\section{References}

Enraf-Nonius (1989). CAD-4 Software. Enraf-Nonius, Delft, The Netherlands.

Harms, K. \& Wocadlo, S. (1995). XCAD4. University of Marburg, Germany. North, A. C. T., Phillips, D. C. \& Mathews, F. S. (1968). Acta Cryst. A24, 351359.

Sheldrick, G. M. (2008). Acta Cryst. A64, 112-122.

Xiao, Z.-P., Fang, R.-Q., Li, H.-Q., Shi, L., Xue, J.-Y., Zheng, Y. \& Zhu, H.-L. (2008). Eur. J. Med. Chem. 43, 1828-1836.

Xiao, Z.-P., Xue, J.-Y., Tan, S.-H., Li, H.-Q. \& Zhu, H.-L. (2007). Bioorg. Med. Chem. 15, 4212-4219. 


\section{supporting information}

Acta Cryst. (2009). E65, o672 [doi:10.1107/S1600536809007089]

\section{(E)-2-(4-Methoxyphenyl)-N-(2-pyridyl)-3-(2-pyridylamino)acrylamide}

\section{Zhu-Ping Xiao, Xiao-Chun Peng and Ying-Chun Wang}

\section{S1. Comment}

An enamine, a tautomer of a Schiff base, shows a high similarity to the corresponding Schiff base in chemical structure which shows diverse biological activities. Our recent work affirmed that enamine, like Schiff base, exhibited high antibacterial activity (Xiao et al., 2007, 2008). We herein report the crystal structure of the title compound, (I).

As shown in Fig. 1, the title compound is an enamine containing a functional group of amide moiety. The title compound consists of an aminoacrylamide moiety and three aromatic ring fragments. The aminoacrylamide moiety, C8, $\mathrm{C} 9, \mathrm{C} 15, \mathrm{~N} 1, \mathrm{~N} 3$ and O2, is nearly coplanar with a mean deviation of $0.023 \AA$, defined as plane I; C10 to C14 and N2 forms a plane with a mean deviation of $0.005 \AA$, defined as plane II; $\mathrm{C} 16$ to $\mathrm{C} 20$ and N4 forms a plane with a mean deviation of $0.008 \AA$, defined as plane III; $\mathrm{C} 2$ to $\mathrm{C} 7$ forms the fourth plane with a mean deviation of $0.005 \AA$, defined as plane IV. Plane II, plane III and plane IV make a dihedral angle of 4.018 (8), 15.66 (12) and 71.22 (9) ${ }^{\circ}$ with plane I, respectively. The bond distance C8-C9 [1.359 (5) $\AA]$ falls in the range of a typical double bond, and C9-N1 bond [1.352 (4) $\AA$ ] is shorter than the standard $\mathrm{C}-\mathrm{N}$ single bond (1.48 $\AA$ ), but longer than a $\mathrm{C}-\mathrm{N}$ double bond (1.28 $\AA$ ). This clearly indicates that the $\mathrm{p}$ orbital of $\mathrm{N} 1$ is conjugated with the $\pi$ molecular orbital of $\mathrm{C} 8-\mathrm{C} 9$ double bond. For the same reason, we speculate that the $\mathrm{p}$ orbital of $\mathrm{N} 1$ is also conjugated with pyridyl group (plane II) and the p orbital of N3 is conjugated with both pyridyl group (plane III) and carboxyl group $(\mathrm{C} 15=\mathrm{O} 2)$. All other double bonds and single bonds in the molecule fall in normal range of bond lengths.

In the crystal of (I), the structure is stabilized by intermolecular interactions including hydrogen bond $\mathrm{N} 1-\mathrm{H} 1 \mathrm{~A} \cdots \mathrm{N} 4$, $\mathrm{C} 11-\mathrm{H} 11 \mathrm{~A} \cdots \mathrm{N} 4$ and $\mathrm{C} 14-\mathrm{H} 14 \mathrm{~A} \cdots \mathrm{O} 2$; details of hydrogen-bond geometry are given in Table 1.

\section{S2. Experimental}

Equimolar quantities ( $6 \mathrm{mmol}$ ) of 2-(4-methoxyphenyl)-3-oxo- $N$-(pyridin-2-yl)propanamide (1.62 g) and 2-aminobenzenamine $(0.56 \mathrm{~g})$ in absolute alcohol $(18 \mathrm{ml})$ were heated at $344-354 \mathrm{~K}$ for $2 \mathrm{~h}$. The excess solvent was removed under reduced pressure. The residue was purified by a flash chromatography with EtOAc-petrolum ether to afford two fractions. The first fraction gave a $Z$-isomer, and the second fraction, after partial solvent evaporation, furnished colourless blocks of (I) suitable for single-crystal structure determination.

\section{S3. Refinement}

All $\mathrm{H}$ atoms were placed in geometrically idealized positions $(\mathrm{C}-\mathrm{H}=0.93-0.96 \AA, \mathrm{N}-\mathrm{H}=0.86 \AA)$ and refined as riding with $U_{\text {iso }}(\mathrm{H})=1.2 \mathrm{U}_{\text {eq }}(\mathrm{C}, \mathrm{N})$ or $1.5 \mathrm{U}_{\mathrm{eq}}($ methyl C). 


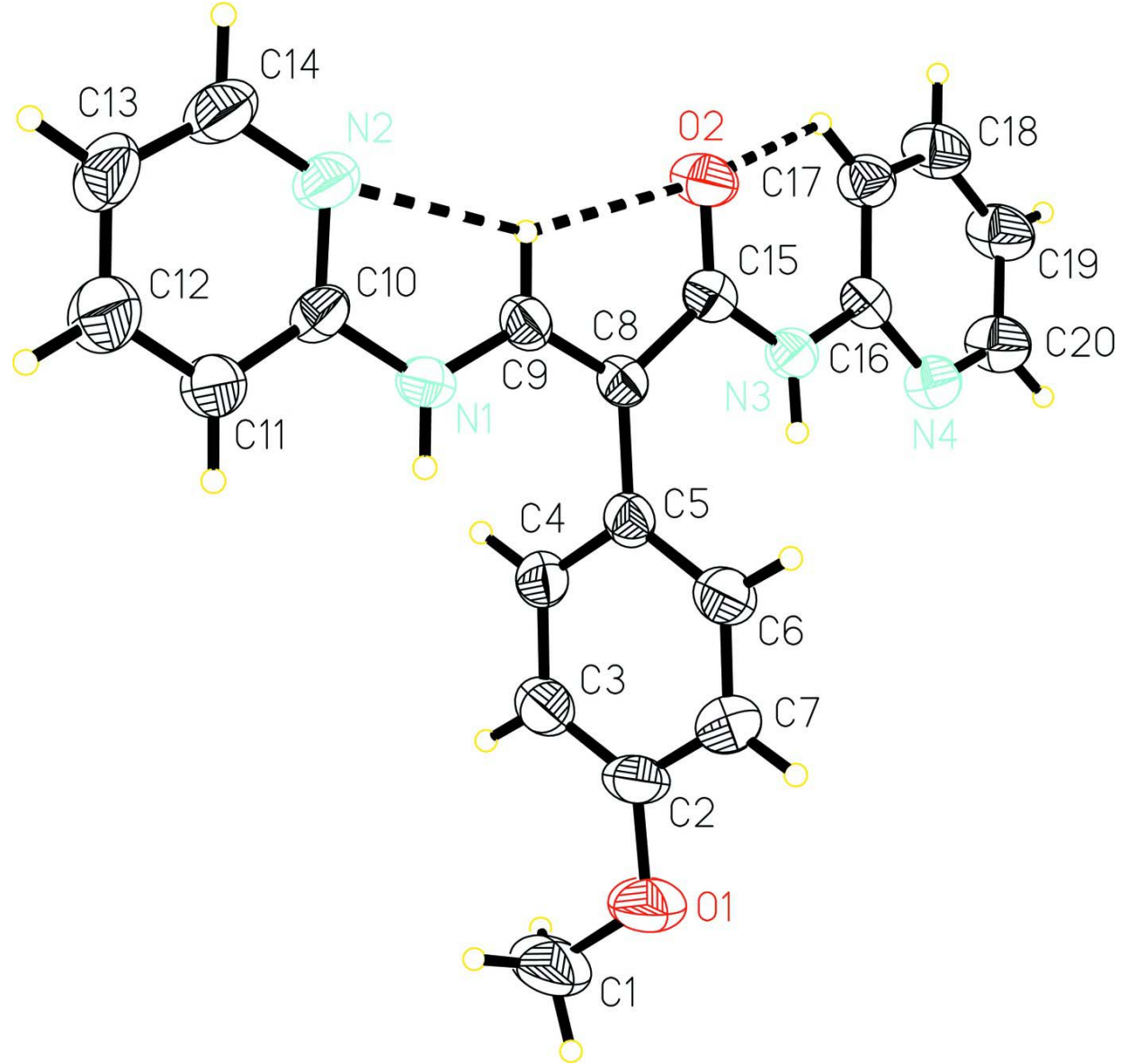

\section{Figure 1}

The molecular structure of (I) with displacement ellipsoids for the non-hydrogen atoms drawn at the $30 \%$ probability level.

\section{(E)-2-(4-Methoxyphenyl)-N-(2-pyridyl)-3-(2-pyridylamino)acrylamide}

\section{Crystal data}

$\mathrm{C}_{20} \mathrm{H}_{18} \mathrm{~N}_{4} \mathrm{O}_{2}$

$M_{r}=346.38$

Monoclinic, $P 2_{1} / c$

Hall symbol: -P 2ybc

$a=11.546$ (2) $\AA$

$b=12.148(2) \AA$

$c=14.006(3) \AA$

$\beta=113.74(3)^{\circ}$

$V=1798.3(6) \AA^{3}$

$Z=4$

\section{Data collection}

Enraf-Nonius CAD-4 diffractometer

Radiation source: fine-focus sealed tube Graphite monochromator $\omega / 2 \theta$ scans
$F(000)=728$

$D_{\mathrm{x}}=1.279 \mathrm{Mg} \mathrm{m}^{-3}$

Mo $K \alpha$ radiation, $\lambda=0.71073 \AA$

Cell parameters from 25 reflections

$\theta=9-12^{\circ}$

$\mu=0.09 \mathrm{~mm}^{-1}$

$T=293 \mathrm{~K}$

Block, colorless

$0.30 \times 0.10 \times 0.10 \mathrm{~mm}$

Absorption correction: $\psi$ scan

(North et al., 1968)

$T_{\min }=0.975, T_{\max }=0.992$

3523 measured reflections

3523 independent reflections 
1452 reflections with $I>2 \sigma(I)$

$R_{\text {int }}=0.000$

$\theta_{\text {max }}=26.0^{\circ}, \theta_{\text {min }}=1.9^{\circ}$

$h=-14 \rightarrow 13$

\section{Refinement}

Refinement on $F^{2}$

Least-squares matrix: full

$R\left[F^{2}>2 \sigma\left(F^{2}\right)\right]=0.083$

$w R\left(F^{2}\right)=0.200$

$S=1.04$

3523 reflections

235 parameters

0 restraints

Primary atom site location: structure-invariant direct methods $k=0 \rightarrow 14$

$l=0 \rightarrow 17$

3 standard reflections every 200 reflections

intensity decay: none

Secondary atom site location: difference Fourier map

Hydrogen site location: inferred from neighbouring sites

$\mathrm{H}$-atom parameters constrained

$w=1 /\left[\sigma^{2}\left(F_{\mathrm{o}}^{2}\right)+(0.0712 P)^{2}\right]$

where $P=\left(F_{\mathrm{o}}{ }^{2}+2 F_{\mathrm{c}}{ }^{2}\right) / 3$

$(\Delta / \sigma)_{\max }<0.001$

$\Delta \rho_{\max }=0.16 \mathrm{e} \AA^{-3}$

$\Delta \rho_{\text {min }}=-0.16$ e $\AA^{-3}$

Special details

Geometry. All e.s.d.'s (except the e.s.d. in the dihedral angle between two 1.s. planes) are estimated using the full covariance matrix. The cell e.s.d.'s are taken into account individually in the estimation of e.s.d.'s in distances, angles and torsion angles; correlations between e.s.d.'s in cell parameters are only used when they are defined by crystal symmetry. An approximate (isotropic) treatment of cell e.s.d.'s is used for estimating e.s.d.'s involving 1.s. planes.

Refinement. Refinement of $F^{2}$ against ALL reflections. The weighted $R$-factor $w R$ and goodness of fit $S$ are based on $F^{2}$, conventional $R$-factors $R$ are based on $F$, with $F$ set to zero for negative $F^{2}$. The threshold expression of $F^{2}>\sigma\left(F^{2}\right)$ is used only for calculating $R$-factors $(\mathrm{gt})$ etc. and is not relevant to the choice of reflections for refinement. $R$-factors based on $F^{2}$ are statistically about twice as large as those based on $F$, and $R$-factors based on ALL data will be even larger.

Fractional atomic coordinates and isotropic or equivalent isotropic displacement parameters $\left(\AA^{2}\right)$

\begin{tabular}{|c|c|c|c|c|}
\hline & $x$ & $y$ & $z$ & $U_{\text {iso }} * / U_{\text {eq }}$ \\
\hline N1 & 0.8670 & $0.3253(3)$ & $0.3474(3)$ & $0.0628(10)$ \\
\hline H1A & 0.9483 & 0.3244 & 0.3725 & $0.075^{*}$ \\
\hline $\mathrm{O} 1$ & $1.3868(3)$ & $0.5123(3)$ & $0.3652(3)$ & $0.1153(14)$ \\
\hline $\mathrm{C} 1$ & $1.4551(5)$ & $0.4266(5)$ & $0.3437(5)$ & $0.137(3)$ \\
\hline H1B & 1.5434 & 0.4450 & 0.3718 & $0.206^{*}$ \\
\hline $\mathrm{H} 1 \mathrm{C}$ & 1.4251 & 0.4168 & 0.2696 & $0.206^{*}$ \\
\hline H1D & 1.4432 & 0.3596 & 0.3750 & $0.206^{*}$ \\
\hline $\mathrm{O} 2$ & $0.6560(2)$ & $0.5617(3)$ & $0.1480(2)$ & $0.0756(9)$ \\
\hline N2 & 0.6813 & $0.2469(3)$ & $0.3418(3)$ & $0.0752(11)$ \\
\hline $\mathrm{C} 2$ & $1.2592(4)$ & $0.5024(5)$ & $0.3320(4)$ & $0.0820(15)$ \\
\hline N3 & $0.8252(3)$ & $0.6425(3)$ & $0.1344(2)$ & $0.0591(9)$ \\
\hline $\mathrm{H} 3 \mathrm{~A}$ & 0.9063 & 0.6471 & 0.1648 & $0.071^{*}$ \\
\hline $\mathrm{C} 3$ & $1.1876(4)$ & $0.4216(4)$ & $0.2676(4)$ & $0.0764(13)$ \\
\hline H3B & 1.2254 & 0.3682 & 0.2420 & $0.092 *$ \\
\hline N4 & 0.8483 & $0.7805(3)$ & 0.0328 & $0.0635(9)$ \\
\hline $\mathrm{C} 4$ & $1.0576(4)$ & $0.4195(3)$ & 0.2404 & $0.0648(11)$ \\
\hline H4B & 1.0096 & 0.3645 & 0.1958 & $0.078^{*}$ \\
\hline $\mathrm{C} 5$ & $0.9974(4)$ & $0.4961(3)$ & 0.2771 & $0.0568(11)$ \\
\hline C6 & $1.0728(4)$ & $0.5774(4)$ & 0.3409 & $0.0743(13)$ \\
\hline H6A & 1.0356 & 0.6313 & 0.3665 & $0.089 *$ \\
\hline $\mathrm{C} 7$ & $1.2003(4)$ & $0.5811(4)$ & $0.3678(4)$ & $0.0874(15)$ \\
\hline
\end{tabular}




$\begin{array}{lllll}\text { H7A } & 1.2479 & 0.6373 & 0.4107 & 0.105^{*} \\ \text { C8 } & 0.8599(3) & 0.4900(3) & 0.2477(3) & 0.0545(10) \\ \text { C9 } & 0.8061(4) & 0.4077(3) & 0.2814(3) & 0.0583(11) \\ \text { H9A } & 0.7183 & 0.4081 & 0.2566 & 0.070^{*} \\ \text { C10 } & 0.8054(4) & 0.2412(3) & 0.3773(3) & 0.0602(11) \\ \text { C11 } & 0.8757(4) & 0.1591(4) & 0.4414(4) & 0.0797(15) \\ \text { H11A } & 0.9634 & 0.1585 & 0.4652 & 0.096^{*} \\ \text { C12 } & 0.8136(5) & 0.0787(4) & 0.4691(4) & 0.1026(18) \\ \text { H12A } & 0.8588 & 0.0216 & 0.5122 & 0.123^{*} \\ \text { C13 } & 0.6855(5) & 0.0816(4) & 0.4340(4) & 0.0955(17) \\ \text { H13A } & 0.6416 & 0.0268 & 0.4518 & 0.115^{*} \\ \text { C14 } & 0.6240(5) & 0.1666(4) & 0.3725(4) & 0.0884(16) \\ \text { H14A } & 0.5365 & 0.1697 & 0.3499 & 0.106^{*} \\ \text { C15 } & 0.7711(4) & 0.5672(3) & 0.1737(3) & 0.0537(10) \\ \text { C16 } & 0.7672(4) & 0.7143(3) & 0.0506(3) & 0.0559(10) \\ \text { C17 } & 0.6370(4) & 0.7165(4) & -0.0105(3) & 0.0671(12) \\ \text { H17A } & 0.5812 & 0.6716 & 0.0045 & 0.080^{*} \\ \text { C18 } & 0.5952(5) & 0.7878(4) & -0.0932(4) & 0.0870(15) \\ \text { H18A } & 0.5094 & 0.7909 & -0.1362 & 0.104^{*} \\ \text { C19 } & 0.6782(5) & 0.8542(4) & -0.1129(4) & 0.0860(16) \\ \text { H19A } & 0.6505 & 0.9018 & -0.1697 & 0.103^{*} \\ \text { C20 } & 0.8027(4) & 0.8489(4) & -0.0471(4) & 0.0811(14) \\ \text { H20A } & 0.8588 & 0.8961 & -0.0591 & 0.097^{*} \\ & & & & \end{array}$

Atomic displacement parameters $\left(\AA^{2}\right)$

\begin{tabular}{lllllll}
\hline & $U^{11}$ & $U^{22}$ & $U^{33}$ & $U^{12}$ & $U^{13}$ & $U^{23}$ \\
\hline $\mathrm{N} 1$ & $0.0472(19)$ & $0.066(2)$ & $0.072(2)$ & $0.0045(18)$ & $0.0203(17)$ & $0.011(2)$ \\
$\mathrm{O} 1$ & $0.056(2)$ & $0.122(3)$ & $0.161(4)$ & $0.003(2)$ & $0.036(2)$ & $0.026(3)$ \\
$\mathrm{C} 1$ & $0.066(3)$ & $0.138(6)$ & $0.215(7)$ & $0.023(4)$ & $0.064(4)$ & $0.051(5)$ \\
$\mathrm{O} 2$ & $0.0566(18)$ & $0.090(2)$ & $0.082(2)$ & $0.0110(16)$ & $0.0303(16)$ & $0.0173(18)$ \\
$\mathrm{N} 2$ & $0.055(2)$ & $0.079(3)$ & $0.086(3)$ & $-0.011(2)$ & $0.023(2)$ & $0.018(2)$ \\
$\mathrm{C} 2$ & $0.046(3)$ & $0.091(4)$ & $0.100(4)$ & $0.005(3)$ & $0.020(3)$ & $0.032(3)$ \\
$\mathrm{N} 3$ & $0.0523(19)$ & $0.056(2)$ & $0.059(2)$ & $0.0026(17)$ & $0.0119(17)$ & $0.0122(18)$ \\
$\mathrm{C} 3$ & $0.070(3)$ & $0.073(3)$ & $0.092(4)$ & $0.011(3)$ & $0.038(3)$ & $0.015(3)$ \\
$\mathrm{N} 4$ & $0.062(2)$ & $0.070(2)$ & $0.056(2)$ & $0.000(2)$ & $0.0210(18)$ & $0.009(2)$ \\
$\mathrm{C} 4$ & $0.065(3)$ & $0.056(3)$ & $0.067(3)$ & $0.000(2)$ & $0.020(2)$ & $0.002(2)$ \\
$\mathrm{C} 5$ & $0.059(2)$ & $0.051(3)$ & $0.054(3)$ & $0.005(2)$ & $0.015(2)$ & $0.005(2)$ \\
$\mathrm{C} 6$ & $0.064(3)$ & $0.077(3)$ & $0.075(3)$ & $0.009(3)$ & $0.021(2)$ & $-0.004(3)$ \\
$\mathrm{C} 7$ & $0.066(3)$ & $0.083(4)$ & $0.094(4)$ & $-0.007(3)$ & $0.013(3)$ & $-0.008(3)$ \\
$\mathrm{C} 8$ & $0.052(2)$ & $0.050(3)$ & $0.057(3)$ & $0.006(2)$ & $0.0169(19)$ & $0.003(2)$ \\
$\mathrm{C} 9$ & $0.056(2)$ & $0.050(2)$ & $0.059(3)$ & $0.007(2)$ & $0.012(2)$ & $0.000(2)$ \\
$\mathrm{C} 10$ & $0.057(3)$ & $0.063(3)$ & $0.057(3)$ & $-0.015(2)$ & $0.019(2)$ & $0.004(2)$ \\
$\mathrm{C} 11$ & $0.066(3)$ & $0.080(3)$ & $0.084(3)$ & $-0.001(3)$ & $0.021(3)$ & $0.034(3)$ \\
$\mathrm{C} 12$ & $0.093(4)$ & $0.098(4)$ & $0.101(4)$ & $-0.008(3)$ & $0.023(3)$ & $0.040(4)$ \\
$\mathrm{C} 13$ & $0.095(4)$ & $0.081(4)$ & $0.095(4)$ & $-0.022(3)$ & $0.023(3)$ & $0.031(3)$ \\
$\mathrm{C} 14$ & $0.067(3)$ & $0.096(4)$ & $0.098(4)$ & $-0.021(3)$ & $0.030(3)$ & $0.014(3)$ \\
$\mathrm{C} 15$ & $0.055(2)$ & $0.059(3)$ & $0.047(2)$ & $0.004(2)$ & $0.021(2)$ & $-0.003(2)$ \\
& & & & &
\end{tabular}


supporting information

\begin{tabular}{lllllll}
$\mathrm{C} 16$ & $0.057(2)$ & $0.056(3)$ & $0.054(3)$ & $0.010(2)$ & $0.022(2)$ & $0.003(2)$ \\
$\mathrm{C} 17$ & $0.060(3)$ & $0.073(3)$ & $0.062(3)$ & $0.003(2)$ & $0.017(2)$ & $0.009(3)$ \\
$\mathrm{C} 18$ & $0.066(3)$ & $0.106(4)$ & $0.068(3)$ & $0.016(3)$ & $0.005(3)$ & $0.017(3)$ \\
$\mathrm{C} 19$ & $0.074(3)$ & $0.103(4)$ & $0.077(4)$ & $0.017(3)$ & $0.025(3)$ & $0.039(3)$ \\
$\mathrm{C} 20$ & $0.075(3)$ & $0.097(4)$ & $0.071(3)$ & $0.006(3)$ & $0.029(3)$ & $0.023(3)$ \\
\hline
\end{tabular}

Geometric parameters $\left(\hat{A},{ }^{\circ}\right)$

\begin{tabular}{|c|c|c|c|}
\hline $\mathrm{N} 1-\mathrm{C} 9$ & $1.352(4)$ & $\mathrm{C} 5-\mathrm{C} 8$ & $1.472(5)$ \\
\hline $\mathrm{N} 1-\mathrm{C} 10$ & $1.402(5)$ & $\mathrm{C} 6-\mathrm{C} 7$ & $1.366(6)$ \\
\hline $\mathrm{N} 1-\mathrm{H} 1 \mathrm{~A}$ & 0.8600 & C6-H6A & 0.9300 \\
\hline $\mathrm{O} 1-\mathrm{C} 2$ & $1.360(5)$ & C7-H7A & 0.9300 \\
\hline $\mathrm{O} 1-\mathrm{C} 1$ & $1.409(6)$ & $\mathrm{C} 8-\mathrm{C} 9$ & $1.359(5)$ \\
\hline $\mathrm{C} 1-\mathrm{H} 1 \mathrm{~B}$ & 0.9600 & $\mathrm{C} 8-\mathrm{C} 15$ & $1.466(5)$ \\
\hline $\mathrm{C} 1-\mathrm{H} 1 \mathrm{C}$ & 0.9600 & C9-H9A & 0.9300 \\
\hline C1-H1D & 0.9600 & $\mathrm{C} 10-\mathrm{C} 11$ & $1.368(5)$ \\
\hline $\mathrm{O} 2-\mathrm{C} 15$ & $1.231(4)$ & $\mathrm{C} 11-\mathrm{C} 12$ & $1.358(6)$ \\
\hline $\mathrm{N} 2-\mathrm{C} 10$ & $1.315(5)$ & $\mathrm{C} 11-\mathrm{H} 11 \mathrm{~A}$ & 0.9300 \\
\hline $\mathrm{N} 2-\mathrm{C} 14$ & $1.343(5)$ & $\mathrm{C} 12-\mathrm{C} 13$ & $1.358(6)$ \\
\hline $\mathrm{C} 2-\mathrm{C} 3$ & $1.365(6)$ & $\mathrm{C} 12-\mathrm{H} 12 \mathrm{~A}$ & 0.9300 \\
\hline $\mathrm{C} 2-\mathrm{C} 7$ & $1.379(6)$ & $\mathrm{C} 13-\mathrm{C} 14$ & $1.348(6)$ \\
\hline $\mathrm{N} 3-\mathrm{C} 15$ & $1.344(5)$ & $\mathrm{C} 13-\mathrm{H} 13 \mathrm{~A}$ & 0.9300 \\
\hline $\mathrm{N} 3-\mathrm{C} 16$ & $1.398(5)$ & $\mathrm{C} 14-\mathrm{H} 14 \mathrm{~A}$ & 0.9300 \\
\hline $\mathrm{N} 3-\mathrm{H} 3 \mathrm{~A}$ & 0.8600 & $\mathrm{C} 16-\mathrm{C} 17$ & $1.400(5)$ \\
\hline $\mathrm{C} 3-\mathrm{C} 4$ & $1.393(5)$ & $\mathrm{C} 17-\mathrm{C} 18$ & $1.368(6)$ \\
\hline $\mathrm{C} 3-\mathrm{H} 3 \mathrm{~B}$ & 0.9300 & C17-H17A & 0.9300 \\
\hline $\mathrm{N} 4-\mathrm{C} 20$ & $1.321(5)$ & $\mathrm{C} 18-\mathrm{C} 19$ & $1.364(6)$ \\
\hline $\mathrm{N} 4-\mathrm{C} 16$ & $1.332(5)$ & $\mathrm{C} 18-\mathrm{H} 18 \mathrm{~A}$ & 0.9300 \\
\hline $\mathrm{C} 4-\mathrm{C} 5$ & $1.379(5)$ & $\mathrm{C} 19-\mathrm{C} 20$ & $1.361(6)$ \\
\hline $\mathrm{C} 4-\mathrm{H} 4 \mathrm{~B}$ & 0.9300 & C19-H19A & 0.9300 \\
\hline $\mathrm{C} 5-\mathrm{C} 6$ & $1.380(5)$ & $\mathrm{C} 20-\mathrm{H} 20 \mathrm{~A}$ & 0.9300 \\
\hline $\mathrm{C} 9-\mathrm{N} 1-\mathrm{C} 10$ & $123.8(3)$ & $\mathrm{N} 1-\mathrm{C} 9-\mathrm{C} 8$ & $126.7(4)$ \\
\hline $\mathrm{C} 9-\mathrm{N} 1-\mathrm{H} 1 \mathrm{~A}$ & 118.1 & $\mathrm{~N} 1-\mathrm{C} 9-\mathrm{H} 9 \mathrm{~A}$ & 116.7 \\
\hline $\mathrm{C} 10-\mathrm{N} 1-\mathrm{H} 1 \mathrm{~A}$ & 118.1 & $\mathrm{C} 8-\mathrm{C} 9-\mathrm{H} 9 \mathrm{~A}$ & 116.7 \\
\hline $\mathrm{C} 2-\mathrm{O} 1-\mathrm{C} 1$ & $119.0(5)$ & $\mathrm{N} 2-\mathrm{C} 10-\mathrm{C} 11$ & $123.7(4)$ \\
\hline $\mathrm{O} 1-\mathrm{C} 1-\mathrm{H} 1 \mathrm{~B}$ & 109.5 & $\mathrm{~N} 2-\mathrm{C} 10-\mathrm{N} 1$ & $117.1(4)$ \\
\hline $\mathrm{O} 1-\mathrm{C} 1-\mathrm{H} 1 \mathrm{C}$ & 109.5 & $\mathrm{C} 11-\mathrm{C} 10-\mathrm{N} 1$ & $119.2(4)$ \\
\hline $\mathrm{H} 1 \mathrm{~B}-\mathrm{C} 1-\mathrm{H} 1 \mathrm{C}$ & 109.5 & $\mathrm{C} 12-\mathrm{C} 11-\mathrm{C} 10$ & $118.0(4)$ \\
\hline $\mathrm{O} 1-\mathrm{C} 1-\mathrm{H} 1 \mathrm{D}$ & 109.5 & $\mathrm{C} 12-\mathrm{C} 11-\mathrm{H} 11 \mathrm{~A}$ & 121.0 \\
\hline $\mathrm{H} 1 \mathrm{~B}-\mathrm{C} 1-\mathrm{H} 1 \mathrm{D}$ & 109.5 & $\mathrm{C} 10-\mathrm{C} 11-\mathrm{H} 11 \mathrm{~A}$ & 121.0 \\
\hline $\mathrm{H} 1 \mathrm{C}-\mathrm{C} 1-\mathrm{H} 1 \mathrm{D}$ & 109.5 & $\mathrm{C} 11-\mathrm{C} 12-\mathrm{C} 13$ & $120.1(5)$ \\
\hline $\mathrm{C} 10-\mathrm{N} 2-\mathrm{C} 14$ & $116.3(4)$ & $\mathrm{C} 11-\mathrm{C} 12-\mathrm{H} 12 \mathrm{~A}$ & 120.0 \\
\hline $\mathrm{O} 1-\mathrm{C} 2-\mathrm{C} 3$ & $125.0(5)$ & $\mathrm{C} 13-\mathrm{C} 12-\mathrm{H} 12 \mathrm{~A}$ & 120.0 \\
\hline $\mathrm{O} 1-\mathrm{C} 2-\mathrm{C} 7$ & $116.1(5)$ & $\mathrm{C} 14-\mathrm{C} 13-\mathrm{C} 12$ & $117.9(5)$ \\
\hline $\mathrm{C} 3-\mathrm{C} 2-\mathrm{C} 7$ & $118.8(4)$ & $\mathrm{C} 14-\mathrm{C} 13-\mathrm{H} 13 \mathrm{~A}$ & 121.0 \\
\hline $\mathrm{C} 15-\mathrm{N} 3-\mathrm{C} 16$ & $128.6(4)$ & $\mathrm{C} 12-\mathrm{C} 13-\mathrm{H} 13 \mathrm{~A}$ & 121.0 \\
\hline $\mathrm{C} 15-\mathrm{N} 3-\mathrm{H} 3 \mathrm{~A}$ & 115.7 & $\mathrm{~N} 2-\mathrm{C} 14-\mathrm{C} 13$ & $124.0(5)$ \\
\hline
\end{tabular}




\begin{tabular}{|c|c|c|c|}
\hline $\mathrm{C} 16-\mathrm{N} 3-\mathrm{H} 3 \mathrm{~A}$ & 115.7 & $\mathrm{~N} 2-\mathrm{C} 14-\mathrm{H} 14 \mathrm{~A}$ & 118.0 \\
\hline $\mathrm{C} 2-\mathrm{C} 3-\mathrm{C} 4$ & $119.6(5)$ & $\mathrm{C} 13-\mathrm{C} 14-\mathrm{H} 14 \mathrm{~A}$ & 118.0 \\
\hline $\mathrm{C} 2-\mathrm{C} 3-\mathrm{H} 3 \mathrm{~B}$ & 120.2 & $\mathrm{O} 2-\mathrm{C} 15-\mathrm{N} 3$ & $122.9(4)$ \\
\hline $\mathrm{C} 4-\mathrm{C} 3-\mathrm{H} 3 \mathrm{~B}$ & 120.2 & $\mathrm{O} 2-\mathrm{C} 15-\mathrm{C} 8$ & $122.5(4)$ \\
\hline $\mathrm{C} 20-\mathrm{N} 4-\mathrm{C} 16$ & $117.9(4)$ & $\mathrm{N} 3-\mathrm{C} 15-\mathrm{C} 8$ & $114.6(4)$ \\
\hline $\mathrm{C} 5-\mathrm{C} 4-\mathrm{C} 3$ & $122.4(4)$ & $\mathrm{N} 4-\mathrm{C} 16-\mathrm{N} 3$ & $113.5(3)$ \\
\hline $\mathrm{C} 5-\mathrm{C} 4-\mathrm{H} 4 \mathrm{~B}$ & 118.8 & $\mathrm{~N} 4-\mathrm{C} 16-\mathrm{C} 17$ & $122.5(4)$ \\
\hline $\mathrm{C} 3-\mathrm{C} 4-\mathrm{H} 4 \mathrm{~B}$ & 118.8 & $\mathrm{~N} 3-\mathrm{C} 16-\mathrm{C} 17$ & $124.0(4)$ \\
\hline $\mathrm{C} 4-\mathrm{C} 5-\mathrm{C} 6$ & $116.3(4)$ & $\mathrm{C} 18-\mathrm{C} 17-\mathrm{C} 16$ & $117.1(4)$ \\
\hline $\mathrm{C} 4-\mathrm{C} 5-\mathrm{C} 8$ & $120.7(4)$ & $\mathrm{C} 18-\mathrm{C} 17-\mathrm{H} 17 \mathrm{~A}$ & 121.4 \\
\hline $\mathrm{C} 6-\mathrm{C} 5-\mathrm{C} 8$ & $123.0(4)$ & $\mathrm{C} 16-\mathrm{C} 17-\mathrm{H} 17 \mathrm{~A}$ & 121.4 \\
\hline $\mathrm{C} 7-\mathrm{C} 6-\mathrm{C} 5$ & $122.0(4)$ & $\mathrm{C} 19-\mathrm{C} 18-\mathrm{C} 17$ & $120.6(4)$ \\
\hline $\mathrm{C} 7-\mathrm{C} 6-\mathrm{H} 6 \mathrm{~A}$ & 119.0 & $\mathrm{C} 19-\mathrm{C} 18-\mathrm{H} 18 \mathrm{~A}$ & 119.7 \\
\hline $\mathrm{C} 5-\mathrm{C} 6-\mathrm{H} 6 \mathrm{~A}$ & 119.0 & $\mathrm{C} 17-\mathrm{C} 18-\mathrm{H} 18 \mathrm{~A}$ & 119.7 \\
\hline $\mathrm{C} 6-\mathrm{C} 7-\mathrm{C} 2$ & $120.8(5)$ & $\mathrm{C} 20-\mathrm{C} 19-\mathrm{C} 18$ & $118.2(5)$ \\
\hline $\mathrm{C} 6-\mathrm{C} 7-\mathrm{H} 7 \mathrm{~A}$ & 119.6 & $\mathrm{C} 20-\mathrm{C} 19-\mathrm{H} 19 \mathrm{~A}$ & 120.9 \\
\hline $\mathrm{C} 2-\mathrm{C} 7-\mathrm{H} 7 \mathrm{~A}$ & 119.6 & $\mathrm{C} 18-\mathrm{C} 19-\mathrm{H} 19 \mathrm{~A}$ & 120.9 \\
\hline $\mathrm{C} 9-\mathrm{C} 8-\mathrm{C} 15$ & $115.4(4)$ & $\mathrm{N} 4-\mathrm{C} 20-\mathrm{C} 19$ & $123.7(5)$ \\
\hline $\mathrm{C} 9-\mathrm{C} 8-\mathrm{C} 5$ & $122.2(4)$ & $\mathrm{N} 4-\mathrm{C} 20-\mathrm{H} 20 \mathrm{~A}$ & 118.1 \\
\hline $\mathrm{C} 15-\mathrm{C} 8-\mathrm{C} 5$ & $122.3(4)$ & $\mathrm{C} 19-\mathrm{C} 20-\mathrm{H} 20 \mathrm{~A}$ & 118.1 \\
\hline $\mathrm{C} 1-\mathrm{O} 1-\mathrm{C} 2-\mathrm{C} 3$ & $-8.5(8)$ & $\mathrm{N} 2-\mathrm{C} 10-\mathrm{C} 11-\mathrm{C} 12$ & $0.5(7)$ \\
\hline $\mathrm{C} 1-\mathrm{O} 1-\mathrm{C} 2-\mathrm{C} 7$ & $171.2(5)$ & $\mathrm{N} 1-\mathrm{C} 10-\mathrm{C} 11-\mathrm{C} 12$ & $-180.0(4)$ \\
\hline $\mathrm{O} 1-\mathrm{C} 2-\mathrm{C} 3-\mathrm{C} 4$ & $179.1(4)$ & $\mathrm{C} 10-\mathrm{C} 11-\mathrm{C} 12-\mathrm{C} 13$ & $-0.4(8)$ \\
\hline $\mathrm{C} 7-\mathrm{C} 2-\mathrm{C} 3-\mathrm{C} 4$ & $-0.6(7)$ & $\mathrm{C} 11-\mathrm{C} 12-\mathrm{C} 13-\mathrm{C} 14$ & $-0.6(9)$ \\
\hline $\mathrm{C} 2-\mathrm{C} 3-\mathrm{C} 4-\mathrm{C} 5$ & $-0.7(7)$ & $\mathrm{C} 10-\mathrm{N} 2-\mathrm{C} 14-\mathrm{C} 13$ & $-1.6(7)$ \\
\hline $\mathrm{C} 3-\mathrm{C} 4-\mathrm{C} 5-\mathrm{C} 6$ & $1.5(6)$ & $\mathrm{C} 12-\mathrm{C} 13-\mathrm{C} 14-\mathrm{N} 2$ & $1.7(9)$ \\
\hline $\mathrm{C} 3-\mathrm{C} 4-\mathrm{C} 5-\mathrm{C} 8$ & $-179.1(4)$ & $\mathrm{C} 16-\mathrm{N} 3-\mathrm{C} 15-\mathrm{O} 2$ & $-10.3(6)$ \\
\hline $\mathrm{C} 4-\mathrm{C} 5-\mathrm{C} 6-\mathrm{C} 7$ & $-1.0(6)$ & $\mathrm{C} 16-\mathrm{N} 3-\mathrm{C} 15-\mathrm{C} 8$ & $168.0(4)$ \\
\hline $\mathrm{C} 8-\mathrm{C} 5-\mathrm{C} 6-\mathrm{C} 7$ & $179.6(4)$ & $\mathrm{C} 9-\mathrm{C} 8-\mathrm{C} 15-\mathrm{O} 2$ & $3.7(6)$ \\
\hline $\mathrm{C} 5-\mathrm{C} 6-\mathrm{C} 7-\mathrm{C} 2$ & $-0.3(7)$ & $\mathrm{C} 5-\mathrm{C} 8-\mathrm{C} 15-\mathrm{O} 2$ & $179.2(4)$ \\
\hline $\mathrm{O} 1-\mathrm{C} 2-\mathrm{C} 7-\mathrm{C} 6$ & $-178.6(4)$ & $\mathrm{C} 9-\mathrm{C} 8-\mathrm{C} 15-\mathrm{N} 3$ & $-174.7(3)$ \\
\hline $\mathrm{C} 3-\mathrm{C} 2-\mathrm{C} 7-\mathrm{C} 6$ & $1.2(7)$ & $\mathrm{C} 5-\mathrm{C} 8-\mathrm{C} 15-\mathrm{N} 3$ & $0.8(5)$ \\
\hline $\mathrm{C} 4-\mathrm{C} 5-\mathrm{C} 8-\mathrm{C} 9$ & $68.4(5)$ & $\mathrm{C} 20-\mathrm{N} 4-\mathrm{C} 16-\mathrm{N} 3$ & $177.8(4)$ \\
\hline $\mathrm{C} 6-\mathrm{C} 5-\mathrm{C} 8-\mathrm{C} 9$ & $-112.2(5)$ & $\mathrm{C} 20-\mathrm{N} 4-\mathrm{C} 16-\mathrm{C} 17$ & $-1.4(6)$ \\
\hline $\mathrm{C} 4-\mathrm{C} 5-\mathrm{C} 8-\mathrm{C} 15$ & $-106.8(4)$ & $\mathrm{C} 15-\mathrm{N} 3-\mathrm{C} 16-\mathrm{N} 4$ & $178.7(4)$ \\
\hline $\mathrm{C} 6-\mathrm{C} 5-\mathrm{C} 8-\mathrm{C} 15$ & $72.6(5)$ & $\mathrm{C} 15-\mathrm{N} 3-\mathrm{C} 16-\mathrm{C} 17$ & $-2.1(6)$ \\
\hline $\mathrm{C} 10-\mathrm{N} 1-\mathrm{C} 9-\mathrm{C} 8$ & $-178.4(4)$ & $\mathrm{N} 4-\mathrm{C} 16-\mathrm{C} 17-\mathrm{C} 18$ & $2.4(6)$ \\
\hline $\mathrm{C} 15-\mathrm{C} 8-\mathrm{C} 9-\mathrm{N} 1$ & $178.4(4)$ & $\mathrm{N} 3-\mathrm{C} 16-\mathrm{C} 17-\mathrm{C} 18$ & $-176.8(4)$ \\
\hline $\mathrm{C} 5-\mathrm{C} 8-\mathrm{C} 9-\mathrm{N} 1$ & $2.9(6)$ & $\mathrm{C} 16-\mathrm{C} 17-\mathrm{C} 18-\mathrm{C} 19$ & $-1.0(7)$ \\
\hline $\mathrm{C} 14-\mathrm{N} 2-\mathrm{C} 10-\mathrm{C} 11$ & $0.5(7)$ & $\mathrm{C} 17-\mathrm{C} 18-\mathrm{C} 19-\mathrm{C} 20$ & $-1.2(8)$ \\
\hline $\mathrm{C} 14-\mathrm{N} 2-\mathrm{C} 10-\mathrm{N} 1$ & $-179.0(4)$ & $\mathrm{C} 16-\mathrm{N} 4-\mathrm{C} 20-\mathrm{C} 19$ & $-1.0(7)$ \\
\hline $\mathrm{C} 9-\mathrm{N} 1-\mathrm{C} 10-\mathrm{N} 2$ & $-3.0(6)$ & $\mathrm{C} 18-\mathrm{C} 19-\mathrm{C} 20-\mathrm{N} 4$ & $2.3(8)$ \\
\hline $\mathrm{C} 9-\mathrm{N} 1-\mathrm{C} 10-\mathrm{C} 11$ & $177.5(4)$ & & \\
\hline
\end{tabular}




\section{supporting information}

Hydrogen-bond geometry $\left(\AA,{ }^{\circ}\right)$

\begin{tabular}{lllll}
\hline$D-\mathrm{H} \cdots A$ & $D-\mathrm{H}$ & $\mathrm{H} \cdots A$ & $D \cdots A$ & $D-\mathrm{H} \cdots A$ \\
\hline $\mathrm{N} 1-\mathrm{H} 1 A \cdots \mathrm{N} 4 \mathrm{i}$ & 0.86 & 2.25 & $3.079(5)$ & 163 \\
$\mathrm{C} 9-\mathrm{H} 9 A \cdots \mathrm{O} 2$ & 0.93 & 2.33 & $2.718(5)$ & 104 \\
$\mathrm{C} 9-\mathrm{H} 9 A \cdots \mathrm{N} 2$ & 0.93 & 2.42 & $2.754(6)$ & 101 \\
$\mathrm{C} 17-\mathrm{H} 17 A \cdots \mathrm{O} 2$ & 0.93 & 2.27 & $2.850(5)$ & 120 \\
$\mathrm{C} 11-\mathrm{H} 11 A \cdots \mathrm{N} 4{ }^{\mathrm{i}}$ & 0.93 & 2.62 & $3.396(6)$ & 141 \\
$\mathrm{C} 14-\mathrm{H} 14 A \cdots \mathrm{O} 2^{\mathrm{ii}}$ & 0.93 & 2.59 & $3.378(5)$ & 143
\end{tabular}

Symmetry codes: (i) $-x+2, y-1 / 2,-z+1 / 2$; (ii) $-x+1, y-1 / 2,-z+1 / 2$. 